WIENER SLAVISTISCHES JAHRBUCH, Band 53/2007, 263-272

(C) 2007 by Österreichische Akademie der Wissenschaften, Wien

GER O F IS C HER

\title{
Perspektiven der Slowenistik am Institut für Slawistik der Universität Wien aus der Sicht der Lehre ${ }^{1}$
}

\begin{abstract}
Slovenistika je stala teko rekoč ob zibelki dunajske slavistike in je kot eden manjših predmetov s sicer že dolgo konstantnim številom študirajočih ostala brez lastne stolice. Dunajska slovenistika danes ni ogrožena zaradi svoje majhnosti, ampak jo ogrožajo bolonjski programi, ki legitimirajo prestrukturiranje oz. standardiziranje visokokega šolstva, kar je spet povezano z likvidiranjem posameznih predmetov in celih študijskih smeri. Na Dunaju je kreativost pri doseganju „,bolonjskih ciljev“ na primer privedla do združitve vseh slavističnih študijskih smeri v eno samo študijsko smer: slavistiko. Za slovenistiko sicer govori, da ima dolgo tradicijo znotraj dunajske slavistike, da gre za jezik avstrijske manjšine in da institut izobražuje tudi učitelje slovenščine za višje šole, vendar glede na trenutne okvirne pogoje prihodnost dunajske slovenistike nikakor ni zagotovljena.
\end{abstract}

In das Zentrum meines Beitrages stelle ich die grundlegende Frage nach der Zukunft der Slowenistik (im weiteren Sinne der Slawistik) an der Universität Wien im Rahmen der derzeit laufenden Reform der Hochschul- und Studienarchitektur und ziehe daraus die entsprechenden Schlüsse.

\section{VORBEMERKUNGEN}

Die Slowenistik stand gewissermaßen an der Wiege der Wiener Slawistik, sie ist bis heute ein eher kleines Fach mit konstanter Studierendenzahl geblieben. Sie stellt - anders als die Russistik, Bohemistik, Polonistik - keine selbständige Lehrkanzel dar. Die slowenistische fachliche Ausbildung wird zu einem großen Teil von Vertre-

1 Dieser Text ist aus der Perspektive eines „Studienprogrammleiters“ geschrieben, einer Funktion, die im Zuge der Neustrukturierung der Universitäten geschaffen wurde und im Wesentlichen mit Organisation und Planung eines Bündels von (inhaltlich mehr oder weniger zusammenhängenden) Studienrichtungen (im konkreten Fall: Slawistik, Fennistik, Hungarologie, Skandinavistik) befasst ist. 
tern der ,allgemeinen Slawistik“, der Südslawistik sowie in ausreichendem Maße durch Gastprofessuren bestritten, für die Sprachausbildung stehen Lektorinnen (fallweise ergänzt durch „Gäste“ aus Slowenien) zur Verfügung. Der Ausbildungsbereich Slowenisch als Lehramt - und nicht zu vergessen der Umstand, dass Slowenisch Minderheitensprache ist - verleiht dem Fach jedoch insgesamt ein (hochschul)politisch nicht unbedeutendes Gewicht. Ob allerdings ein eigenes Slowenistik-Ordinariat am Wiener Institut für Slawistik angesichts der verlaufenden und anstehenden organisatorischen Umstrukturierungen der Hochschulstudien dem Fach sichere $\mathrm{Zu}$ kunftsperspektiven garantieren könnte (bzw. hätte können), ist, wie wir noch sehen werden, durchaus fraglich. Durch die Universitätsreformen der letzten Jahre wurden die Kriterien für die Existenzbedingungen von Studienrichtungen grundlegend verändert, betriebswirtschaftliche Philosophien und harte Kalkulation geben nun das Muster vor, nach dem sich die Studien ohne Ausnahme zu richten haben. In Deutschland und der Schweiz wurde und wird vorgeführt, wie mit Studien und Instituten verfahren wird, die den geforderten Vorgaben nicht entsprechen. Es gibt zur Genüge Beispiele, wo Slawistikinstitute und slawistische Studienangebote liquidiert oder mit anderen fusioniert wurden und werden. Auch Österreich kann hier mithalten: An der Universität Innsbruck wurde im Vorjahr handstreichartig von der Universitätsleitung das Lehramtstudium für Russisch (und andere Fächer) gekappt, zu Beginn der Sommerferien 2006 strauchelten jedoch an der Universität Graz der Dekan und der Forschungsdekan beim Versuch, die Fächer Slawistik (minus Russisch), Klassische Philologie, Alte Geschichte und Archäologie und Südosteuropageschichte zu einem „Balkaninstitut“ im Zuge einer „Neuausrichtung der Geisteswissenschaften“ zu vereinen (die Initiatoren legten auf Grund der massiven Proteste ihre Ämter nieder). Bei diesen Beispielen fühlt man sich an die in Großkonzernen herrschenden zur Normalität gewordenen unsensiblen bis brutalen Managementstile erinnert.

Auch wenn diese Beispiele als Einzelfälle gehandelt werden mögen, kann die Situation nicht als kritisch genug eingeschätzt werden, es ist zu offensichtlich geworden, dass (auch in anderen europäischen Ländern) Gesetzgeber und Universitätsleitungen ein bestimmtes bisher erreichtes Lehrangebot nicht weiter aufrecht erhalten und es zu Gunsten anderer Studien(richtungen) auflösen bzw. deren Ressourcen umverteilen wollen. Dass diese Veränderungen ohne inhaltliche Auseinandersetzung und ohne Einbeziehung der betroffenen Fächer geschehen, gehört zum neuen Stil der „Universität-Neu“. Da der Begehrlichkeiten anderer, in der Regel ebenfalls unterdotierter Fachrichtungen naturgemäß viele sind, kommt Solidarität nicht groß auf.

Der Universitätshistoriker Weber umreißt die Hauptstoßrichtung der aktuellen Entwicklung der europäischen Universität folgendermaßen:

Die stärkste Tendenz der jüngsten Universitätsgeschichte zielt auf eine Begrenzung des universitären Horizonts auf die Gegenwart. Sämtliche Reformansätze orientieren sich ebenfalls an den Bedürfnissen der Gegenwart, während jede ernst zu nehmende zivilisatorisch-humanistische Selbstreflexion und Normenverständigung notwendig Vergangenheit, Gegenwart und Zukunft ins Kalkül ziehen muss. Derartige, für den menschheitlichen Fortschritt unerlässliche Selbstreflexion kann genau aus diesem Grunde keineswegs den Medien überlassen werden, wie viele Universitätskritiker fälschlich meinen. Denn gerade 
die Medien sind - und zwar extrem - in ihrem Horizont gegenwartsbezogen, tagesaktuell und damit notwendig flüchtig und oberflächlich. Mit anderen Worten, die von der Universität direkt und indirekt hervorgebrachte, institutionalisierte gesellschaftlich-kulturell-politische kritische Selbstreflexion, die Europa universalhistorisch so bedeutsam gemacht hat, bedarf auch künftig einer institutionellen, relativ gegenwartsbezogenen, freien und glaubwürdigen Basis. Dieser Aspekt findet in der aktuellen Diskussion offenkundig noch nicht seine angemessene Würdigung. (Weber 2002: 244 f.)

Und an anderer Stelle meint der Autor, dass eine

Transformation der Universität zur ökonomisierten Fach- und Projektuniversität der ,neuen Wissensgesellschaft‘ (Weber 2002: 236)

zu erwarten sei.

Die sog. „Entlassung der Universitäten in die Selbständigkeit“ hat den inneruniversitären Verteilungskampf um Ressourcen und Einfluss dermaßen verschärft, dass immer öfter die Universitätsleitungen (bzw. das Universitätsmanagement) ,nicht erfolgreichen" Fächern die Rute ins Fenster stellen, indem sie mit der Liquidierung bzw. Auflösung der Fächer drohen, die keinen betriebswirtschaftlich argumentierten „Erfolg“ nachweisen können. Als dessen Maßzahl gilt in der Regel das quantitative Verhältnis der Absolventen zu den Studienanfängern. Dabei werden Wirtschaftlichkeits- und Effizienzkriterien einheitlich an die Studienrichtungen angelegt, ohne Rücksicht auf die in ihnen herrschenden unterschiedlichen und oft nicht vergleichbaren Bedingungen. Der Mainstream der Universitätsreformer und der veröffentlichten Meinung sieht zudem die geisteswissenschaftlichen Fächer als hypertroph und gesellschaftlich (d. h. gleichgesetzt mit ökonomisch) unnütz an, und so scheint es politisch kein Problem zu sein, im Gleichklang mit (kaum hinterfragten) neoliberal getönten Anwürfen („Ineffizienz der Wissensvermittlung“, ,,aufgeblähter Ballast an unzeitgemäßem und überflüssigem Bildungswissen“ und dergleichen) die geistesund kulturwissenschaftlichen Fächer generell in die Mangel nehmen zu können. Diese Sichtweise hat auch letztendlich zur folgenreichen Entscheidung der europäischen Hochschulpolitiker geführt, dass die europäische Universitätsreform sich am US-amerikanischen Hochschulmodell zu orientieren habe.

Mit einem organisatorischen Instrumentarium, wie es bei der Sanierung von Industriebetrieben angewandt wird, sollen nun die Hohen Schulen neu strukturiert werden, beginnend mit dem Umbau bzw. der Zerschlagung der demokratischen Selbstverwaltungsstrukturen und endend bei der Neuordnung von Studienrichtungen und Lehrinhalten. Dabei soll die Universität „,schlanker“, „,effizienter“, „zeitgemäßer" werden, es sollen allerorten „Synergieeffekte“ geschaffen und genutzt werden. Die „Universität-Neu“ wird dann eine Wirtschaftskonzernen ähnliche interne Struktur haben mit einem Management an der Spitze, das keiner demokratisch legitimierten Kontrolle auf den unteren Ebenen (wie entscheidungsbefugte Fakultätskollegien, Institute) unterliegt. Das „Studium-Neu“ in Form der „Bologna-Architektur“ soll sich durch eine entsprechende klare Struktur auszeichnen und sich auch inhaltlich (stärker) am Arbeitsmarkt orientieren. Zugangsverfahren, Aufnahmebeschränkungen, präzise definierte Curricula, ein System von Evaluationen, Verschärfungen 
von Prüfungen etc. sollen das Verhältnis von „Input“ und „Output“ in einer ökonomisch legitimierten Balance halten. Zudem halten diverse Sanktionsmechanismen (im Wesentlichen Zuteilung von finanziellen Ressourcen) Institute (bzw. „Organisationseinheiten") auf Linie, d. h. sie rauben ihnen in Wirklichkeit fast jede Gestaltungsfreiheit.

Die „Bologna-Architektur“ sieht vor, dass in einem Grundstudium (Bakkalaureat, sechs Semester) in erster Linie das Wissen vermittelt werden soll, das direkt zu berufsbefähigenden Abschlüssen führt. Erweiterungen und Vertiefungen der Wissensvermittlung, des Wissenserwerbs sind in einem entsprechenden Vertiefungsstudium (MA / Master-Studium, zwei Jahre), bzw. in verschiedenen postgradualen Lehrgängen möglich. Den dritten Teil dieser Studienarchitektur bildet dann das Doktoratsstudium, das vorwiegend dem wissenschaftlichen Nachwuchs dient. Allfällige pädagogische Mehrleistungen sollen nicht durch Aufstockung des Lehrpersonals erzielt sondern „kostenneutral“ erbracht werden, das kann auch heißen durch Erhöhung der Lehrverpflichtung (hier kann die Tschechische Republik als „Vorbild“ gelten mit absurd hohen Lehrverpflichtungen von 20 und mehr Stunden pro Woche), mit Planzielvorgaben und Regulierungen, die in ihrer Hybris realsozialistischen Mustern kaum nachstehen (eine Analyse der Sprache, der Umgangsformen der neuen hierarchischen Strukturen, etc. wäre ein spannendes Thema für sich). Dadurch werden die Arbeitsbedingungen insbesondere der Lektorinnen und Lektoren, der Assistentinnen und Assistenten ebenso wie Karrierechancen nach dem Diktat der „Kostenneutralität" ausgerichtet, der Leistungs- und Konkurrenzdruck wird enorm gesteigert. Unter dem Slogan einer ,zeitgemäßen Pädagogik“ wird die Auslagerung der Lehre auf Medien forciert (darunter auch E-Learning), wofür zusätzliche finanzielle Mittel bereitgestellt werden - unabhängig davon, ob dies im Einzelfall didaktisch sinnvoll ist oder nicht.

Da die inneruniversitären demokratischen Plattformen (im Zuge der „Entschlackung“ der universitären Strukturen) abgeschafft wurden, findet innerhalb der Universität bzw. der Institute auch kaum ein öffentlicher und vor allem entscheidungsrelevanter Diskurs über die Zukunft der Universitäten, über Inhalt und Form der Lehre, die Verbindung von Forschung und Lehre usw. mehr statt. Das Universitätsmanagement kann autoritär, d. h. ohne Anhörung der Betroffenen, bestimmen, welche Studienrichtungen es für ,unrentabel“ hält, wie viele Studien ein Institut anbieten darf, wo in Zukunft Geld investiert wird und dergleichen. Wenn Studienrichtungen aus dem bisherigen Angebot verschwinden, dann überantwortet man den Studierenden, sich entsprechend flexibel zu zeigen und dort hin zu gehen, wo es das vor Ort ,abgewickelte“ Studienangebot noch gibt. Auf diese Weise verlagern Regierungen die Folgen ihrer bildungspolitischen Entscheidungen - Liquidierung von Studienrichtungen, Weigerung des Ausbaus überfüllter Studien etc. - auf andere Länder, d. h. im Grunde lagern sie sie auf Kosten dieser anderen Länder aus. Die Liquidierung von Fächern und ihr Transfer auf andere Standorte haben zur Folge, dass von der alten universitas - der Wissensvielfalt, der Vielfalt der Diskurse - an den derart 
leergeräumten und effektuierten, ,verschlankten“ Universitäten nicht mehr viel übrig bleibt. Es wäre kein Wunder, wenn am Ende des Bologna-Prozesses vor allem berufsspezifische Lehrgänge mit engem Qualifikationsprofil und standardisierten Curricula die Universitäten dominierten. Ich halte es für absurd aber leider für gar nicht ausgeschlossen, dass als Folge der Vereinheitlichungsmanie europaweit einheitliche Curricula, Lehrmaterialien, Medien und sogar Prüfungsverfahren entwickelt und realisiert werden könnten, nur weil sie ökonomisch durchaus begründbar wären. Dann könnte es auch einheitliche Prüfungstermine und eine zentrale computergesteuerte Leistungsbeurteilung geben. Damit wäre nicht nur der Reformprozess an sein Ende gekommen sondern, auch jegliche akademische Tradition von Wissensvermittlung und wohl auch von akademischem Diskurs. Sollte der „Bologna-Prozess“ als Ironie der Geschichte das Ende der Entwicklung der Universität darstellen, die in Bologna (offizielles Gründungsdatum der ersten Universität Europas 1088) ihren Anfang genommen hat?

Nun, lassen wir uns von den Negativutopien nicht allzu schrecken, neben dem Mainstream formiert sich zunehmend Dissens, auch wenn er bisher allerdings kaum Folgen zeitigt. Die Argumente des Dissenses stellen Inhalt und Qualität des Studiums in den Vordergrund - was im Diskurs der offiziellen Hochschulpolitik entweder in unbegründbaren Euphemismen eingepackt oder überhaupt ausgeblendet wird. „Denn das sind die Befunde: Die kürzeren Bachelor-Studien zwingen oft zur Einschränkung des Stoffes, dabei bedeutet Berufsfähigkeit für Geisteswissenschaftler zumeist, vielfältige Fähigkeiten zu haben“ (Kaube 2006). Diese Probleme tauchen aber auch massiv in völlig anderen Bereichen (auch an Fachhochschulen/FH) auf und lassen sich analog auf unsere Fächer umlegen, folgen wir also Rolf Nobel, Professor für Fotografie am Fachbereich Design und Medien der FH Hannover, der feststellt:

Gehen wir davon aus, dass die Qualität der eigenen Arbeit von wesentlicher Bedeutung dafür ist, ob man sich am Markt durchsetzen kann, dann muss eine gute Hochschulausbildung deren Grundlage sein. Verkürzt man die aber bei vielen FHs und Hochschulen gleich um drei Semester, dann vermag ich nicht zu sehen, wie man die bisherige Qualität halten kann. Im Umkehrschluss hieße es nämlich, dass Professoren und Studenten im länger dauernden Diplom-Studium Zeit unnötig vertrödelt hätten. (Nobel 2003)

Er spricht sich massiv gegen ein „Überflieger-Studium von nur 6 Semestern“ aus:

[...] das gerade mal der Dauer einer handwerklichen Ausbildung in der Fotografie entspricht. [...] Mit der Verschulung des Studiums hat die Präsenzpflicht der Studenten nun ein $\mathrm{Ma} \beta$ erreicht, in dem die Studierenden kaum noch nebenbei arbeiten können. [...] kein vernünftiges Fördersystem [...], das es allen Studenten ermöglicht, ohne Zeit raubendes Jobben auszukommen [...] das Studieren wird wieder eine Frage sozialer Herkunft. [...] Davon abgesehen verhindert das enge Zeitkorsett des neuen Systems, was gerade in der Fotografie von großer Bedeutung ist: das Heranwachsen von Persönlichkeiten, die den vielschichtigen Trugbildern und ethischen Verirrungen der Medienwelt gegenüber gewappnet sind. Das aber kann man nicht im Stundenplan-Rhythmus und mit Modul-Bausteinen erlernen. Vielmehr wird das Studieren unter diesem Lerndruck zum bloßen Konsum von Lehrstoff führen und nicht zu fundierter Auseinandersetzung mit dem Medium. Das Ergebnis sind mediale Analphabeten, von denen es in unserer Mediengesellschaft ohnehin schon zu viele gibt. (Nobel 2003) 


\section{Auswirkungen DES Bologna-PROZESSES AUf DIE SLAWISTIK} UND IM BESONDEREN AUF DIE SLOWENISTIK

In Wien ist die Zahl der Studierenden der Slowenistik schon über viele Jahre konstant, das Potenzial an Interessenten ist weitgehend ausgeschöpft, nennenswerte Steigerungen sind nicht zu erwarten. Geringe Wachstumsprognosen sind auch für das Bulgarische und Ukrainische anzusetzen. Das Slowakische gehört derzeit noch zu den ganz „kleinen“ Studienrichtungen, aber das Potenzial an Studierenden ist noch nicht erschlossen.

Derzeit soll laut Entwicklungsplan der Universität Wien ein einheitliches slawistisches sechs-semestriges Bakkalaureat mit entprechenden „Binnendifferenzierungen" eingerichtet werden, die den bisherigen acht Studienrichtungen entsprechen sollen, und darauf sollen acht vier-semestrige MA-Studiengänge aufbauen. In einer slawistischen Studienrichtung ist die für das Bakkalaureat vorgesehene Studienzeit zu kurz, als dass mehr als die grundlegenden Sprach- und Kulturkenntnisse erworben werden können. In diesem dreijährigen Studium ist das Erreichen der Sprachfertigkeitsstufe C1 (nach dem Europäischen Referenzrahmen) in einer slawischen Sprache (ohne Vorkenntnisse) reichlich illusorisch - und für den Einstieg in ein Berufsleben mit philologischem Hintergrund (Auslandskorrespondenz, Bibliotheksoder Verlagswesen, Übersetzertätigkeit etc.) eindeutig zu wenig. Die Verkürzung des Studiums kann nicht durch irgendwelche „hocheffiziente Spezialdidaktiken“ (die es im Übrigen nicht gibt) kompensiert werden. Der Einwand, dass das BA-Studium Fachanalphabeten und unfertige Persönlichkeiten produziert, ist somit nicht leicht von der Hand zu weisen. Gerade Geisteswissenschaftler müssen, um ihre Chance auf dem Arbeitsmarkt zu wahren, Vielseitigkeit (z. B. allfällige berufsspezifische Qualifikationen wie Medienpraxis, Praxis im Wissenschafts-, Projekt- und Kulturmanagement, Teamfähigkeit, sprachliche und kulturelle Kommunikationskompetenz, etc.) anstreben, dies verlangt aber ausreichend Zeit, die jedoch in den neuen durch die Studienreformen gerafften und auf Effizienz (wie immer sie auch definiert wird) getrimmten, „,verschlankten“ Studiengänge nicht vorgesehen ist. Die aktuelle Studienreform huldigt dem Fetisch der Geschwindigkeit, in der ein Studium „durchgezogen“ wird. Dieses Tempobolzen in Sachen Studium als Wert an sich steht im öffentlichen Diskurs hoch im Kurs, wogegen Fragen der Qualität und der Inhalte des Studiums, die Frage der Persönlichkeitsbildung, die sozioökonomischen Gegebenheiten, unter denen ein Großteil der Studierenden ihr Studium zu bewältigen haben, nur eine untergeordnete Rolle spielen. Tatsache ist jedenfalls, dass die Studierenden eingezwängt in das enge Korsett verschulter Curricula viel zu wenig Freiraum haben, sich neben dem Studium auf andere (wie die oben erwähnten berufsfördernden) Erfahrungen, vor allem aber auch auf Auslandsaufenthalte und andere Praxisfelder einzulassen.

Es gibt jedoch Gruppen von Studierenden, denen die BA-Kurzstudien entgegen kommen. Es handelt sich um Personen, die ein slawistisches Fach im Zweit- bzw. Doppelstudium absolvieren wollen, weil sie (als Angehörige der zweiten Generation 
von Migrantinnen und Migranten oder als Minderheitenangehörige) die Zielsprache schon beherrschen bzw. wesentliche Vorkenntnisse besitzen und mit einem weiteren (leicht zu erwerbenden) akademischen Titel ihr Image aufpolieren wollen. Da nun auch Doppelstudien durch die neuen Studiengesetze erleichtert wurden, werden diese sich auch in den Statistiken niederschlagen und andernorts als erfolgreiche Hochschulpolitik verkauft werden. Insgesamt werden die BA-Studien auf jeden Fall die Absolventenstatistiken und damit die Akademikerquote verbessern, da in Österreich nicht die Zahl der Akademiker sondern die Zahl der Abschlüsse gerechnet wird. Auf diese Weise wird das slawistische BA als „Nebenstudium“ in dem Ausmaß an Image verlieren, wie es gleichzeitig an Beliebtheit (ein vorderer Platz im „Beliebtheits-Ranking" der Studierenden) bei bestimmten Zielgruppen gewinnen wird. Der Effekt könnte sein, dass die Slawistikinstitute mit ihrem BA den eher zweifelhaften Ruf als Produktionsstätte von akademischen Leichtgewichten erwerben.

Wenn dem Konzept nach das BA eher allgemeine bzw. vergleichende Einblicke in das Fach vermitteln soll, so haben die zweijährigen weiterführenden MA-Studien die entsprechenden sprachlichen und fachlichen Kompetenzen zu vermitteln, die im BA nicht entwickelt wurden, und sollen darüber hinaus zu fachlich hochwertigen Abschlüssen führen - eine hohe, schwer einzulösende Auflage. Dies gilt zumindest für das Gros der Studierenden, die Slawistik ohne entsprechende sprachlichen Vorkenntnisse (die keine Zulassungsvoraussetzung sind) zu studieren begonnen haben. $\mathrm{Ob}$ unter diesen Bedingungen das im Rahmen der bisherigen Diplomstudien erreichte Niveau im MA-Studium gehalten werden kann ist mehr als fraglich. Es sei denn, das MA-Studium wird zunehmend (und ausschließlich) von sog. „Muttersprachlern“ gewählt, die entsprechende Startvorteile in der sprachlichen und kulturellen Kompetenz „mitbringen“, was umgekehrt auf eine de facto Diskriminierung der Studierenden ohne einschlägige Vorbildung hinausläuft. Die Auswirkungen auf die MA-Studierendenzahlen könnten fatal sein, beim MA-Studium wäre mit großen Einbrüchen zu rechnen, mit fatalen Konsequenzen für den Weiterbestand der einzelnen MA-Studien. Dieser ist nur gesichert, wenn es den Slawistikinstituten gelingt, innerhalb der gegebenen Rahmenbedingungen den Spagat zu schaffen - die Diskrepanz zwischen der Erreichung des geforderten Niveaus und den Eingangsvoraussetzungen der Studierenden.

Von der Bologna-Architektur erwarten sich die Universitätsreformer unter kaum vorgehaltener Hand noch einen weiteren erheblichen Einsparungseffekt: Sie nehmen an, dass (mindestens) zwischen 20 und 40\% der Studierenden nach dem Bakkalaureat nicht mehr weiter studieren und vielmehr einen Beruf ergreifen werden. Dadurch, so wird erwartet, wird das weiterführende MA-Studium in der Tat auch billiger (man braucht weniger Lehrveranstaltungen und weniger Lehrer, weniger Betreuer, weniger sonstige Mittel). Die in Diskussion stehenden erhöhten Studiengebühren für die MA-Studien werden den sozialen und finanziellen Druck auf die Studierenden ebenfalls steigern (wir müssen davon ausgehen, dass sich etwa $60 \%$ der Studierenden ihr Studium durch Arbeit finanzieren müssen). $\mathrm{Ob}$ und in welchem Ausmaß 
MA-Studien speziell aus Slowenisch (und anderen „kleinen“ Sprachen) von den Studierenden unter diesen Bedingungen überhaupt gewählt werden, bleibt eine große Unbekannte. Der ökonomische Zwang, sich nur mit „,arbeitsmarktgängigen“, „,zukunftsfähigen" Studien zu befassen, wäre dann noch größer - schließlich müssen sich die investierten Studiengebühren ja auch einmal „,rechnen“. Die Folge wäre, dass ausgebildete Spezialisten in diesen bedrohten Fachbereichen (in den Medien, im Kulturmanagement, in der Wirtschaft etc.) zur Rarität würden. Es muss klar festgehalten werden, dass bisher die Slawistikinstitute keineswegs Heerscharen von Arbeitslosen produziert haben, vielmehr sind beschäftigungslose Slawisten eine statistisch nicht relevante Größe.

Bei stagnierender oder rückläufiger Nachfrage werden die entsprechenden MAStudiengänge auf die Dauer auch kein entsprechendes Lehrangebot entwickeln können, denn um die finanziellen Ressourcen gibt es, wie schon skizziert, heftige Konkurrenz. Bei den „kleinen“ Studienrichtungen kann sich diese Entwicklung prekär auswirken angesichts der effizienzorientierten Evaluationen: Die Gefahr, nicht bestimmten quantitativ definierten Hörer-Absolventen-Kennziffern zu genügen, ist groß. Eine sich anbietende Lösung könnte sein, derart gefährdete Fächer mit Nachbarfächern zu „fusionieren“. Für die Wiener Slawistik könnte es unter diesen Umständen notwendig werden, dass derart „gefährdete“ MA-Studien wie Slowenisch und Bulgarisch mit Bosnisch/Kroatisch/Serbisch (B/K/S) (aber auch Ukrainisch mit Russisch und Slowakisch mit Tschechisch) zusammengelegt werden müssen - wenn sie nicht gänzlich verschwinden sollen. Diese derart fusionierten Fächer würden wohl die Lehre vereinheitlichen (nicht jedoch sogleich auch die Forschung, da das nach Fächern spezialisierte Personal - noch - vorhanden ist). Die Curricula müssten dem Einsparungsdruck gehorchend für die fusionierten Fächer die Zahl der gemeinsamen Lehrveranstaltungen erhöhen, was auf Kosten der notwendigen Spezialisierung ginge. Der Effekt wäre dann wohl der, dass das MA-Studium mit dem Schwerpunkt sagen wir „Südslawistik“ (also etwa die Fusion aus Slowenistik, Bulgaristik und $\mathrm{B} / \mathrm{K} / \mathrm{S}$ ) kaum viel mehr als sprachliche Grundkenntnisse und einen allgemeinen Überblick über Literatur- und Sprachwissenschaft der südslawischen Sprachen vermitteln können würde und dem Standard nach nicht mehr vergleichbar sein wird wie die ,alten“ Diplomstudien Slowenistik, B/K/S, Bulgaristik. Ob die künftigen Absolventen gegenüber dem bisherigen Studium von Einzelfächern eine größere Breite und Interdisziplinarität gewinnen und ob diese Qualifikationen - wenn sie je realistisch erreichbar sind - ,arbeitsmarktfähiger“ sein werden, mag mehr als bezweifelt werden. Am Arbeitsmarkt werden Fachkräfte gebraucht, die in der Tat über hohe sprachliche und kulturelle Kompetenzen in den konkreten Philologien (also Slowenisch, BKS, Bulgarisch etc.) verfügen - wie übrigens in der Regel bisher die Absolventen des Diplomstudiums.

Ein Thema blieb bisher ausgespart, das Lehramtsstudium. Bisher ist nicht klar, ob und in wie weit dieses in die Bologna-Architektur eingepasst werden soll (notwendig und besonders sinnvoll wäre es nicht). Klar ist jedoch, dass Lehramtskandi- 
daten zur Berufsbefähigung das MA-Niveau erreichen müssen. Bisher war hinsichtlich der erreichten fachlichen Standards das Lehramt mit dem Diplomstudium gleichwertig. Ob dieses Niveau auch beim MA bzw. Lehramt-Neu unter den Bedingungen der neuen „Studienarchitektur“ gehalten/erreicht werden kann, bleibt abzuwarten.

\section{SCHLUSSBEMERKUNGEN}

Die Zukunft der Slowenistik an der Wiener Slawistik ist angesichts der derzeitig herrschenden Bedingungen keineswegs gesichert. Es steht für mich außer Zweifel, dass es viel größerer und resoluterer Anstrengungen als bisher bedarf, den gegebenen Qualitätsstandard der Studienangebote zu erhalten und weiter zu entwickeln. Allerdings haben sich die Voraussetzungen diesen Kampf zu führen durch die Liquidierung der universitären demokratischen Mitbestimmung grundlegend verschlechtert. Die Neuordnung der Universitäten hat zu einer erheblichen Verschlechterung des Betriebsklimas geführt. Mitarbeitern, die immer wieder mit unnachvollziehbaren Entscheidungen zentraler Stellen vor den Kopf gestoßen werden und von fachlicher Mitsprache ausgeschlossen sind, wird dadurch jede Motivation geraubt (Analogien mit Erfahrungen aus der kommunistischen Ära drängen sich vielleicht politisch unkorrekt auf). Planungssicherheit und Transparenz der Entscheidungsgrundlagen bleiben oft auf der Strecke.

Es steht aber noch etwas anderes zur Disposition: Die Verdrängung (und in letzter Konsequenz Liquidierung) slawistischer Studienangebote kollidiert exemplarisch mit dem rhetorisch viel beschworenen integrativen Projekt der EU-Osterweiterung, dem Ziel der Überwindung der jahrzehntelangen Spaltung Europas. Wenn nun das slawistische Studienangebot als für diese politische Aufgabe eminent wichtige Fach reduziert wird, dann stärkt diese Praxis zusätzlich die Argumentation, wonach die EU-Osterweiterung bloß eine Form des Neokolonialismus darstellt (vgl. Hofbauer 2003).

Wenn Studienrichtungen aus dem Lehrangebot verschwinden, dann verarmt der interdisziplinäre Dialog an den betroffenen Universitäten. Was die Slowenistik betrifft, so sind es zwei Gründe, die die Situation für dieses Fach möglicherweise als weniger unmittelbar bedrohlich darstellen: Der Status einer Minderheitensprache und der Status als Lehrfach - aber eine Sicherheitsgarantie sind sie nicht.

Und nicht zuletzt noch ein Wort zu den Hauptbetroffenen - d. h. besser gesagt wohl den Geschädigten - der Studienreformen, den Studierenden: Sie müssen gegenüber früheren Generationen einen höheren Preis für eine mindere Qualität (ihrer Ausbildung) bezahlen. Diesen Skandal hat die herrschende Bildungs- und Hochschulpolitik zu verantworten. 
Hofbauer 2003: H. Hofbauer, Osterweiterung. Vom Drang nach Osten zur peripheren Kaube 2006: EU-Integration, Wien

Kaube 2006: $\quad$ J.Kaube, Befreiungsschlag: Was der Wissenschaftsrat empfiehlt. „Mut Liessmann 2006:

Nobel 2003: zum Abriss der Reformfassade.", FAZ v. 28. 1. 2006

Weber 2002: K. P. Liessmann, Theorie der Unbildung, Wien 2006

R. Nobel, Bin ich im falschen Film?, Photonews 3, 21

W. E. J. Weber, Geschichte der europäischen Universität, Stuttgart

\author{
Gero Fischer \\ Institut für Slawistik der Universität Wien \\ Universitätscampus AAKH, Hof 3 \\ Spitalgasse 2, A-1090 Wien \\ gero.fischer@univie.ac.at
}

